\title{
Cochlear Implant Hearing Rehabilitation in Elderly Patients
}

\author{
Susana Andrade ${ }^{1 *}$ and Jorge Humberto Martins ${ }^{2}$ \\ ${ }^{1}$ ENT Specialist, Otorhinolaryngology Service - Centro Hospitalar de Coimbra, \\ E.P.E., Portugal \\ ${ }^{2}$ Audiologist at ENT Department of University Hospital Center of Coimbra, E.P.E., \\ Portugal \\ *Corresponding Author: Susana Andrade, ENT Specialist, Otorhinolaryngology \\ Service - Centro Hospitalar de Coimbra, E.P.E., Portugal.
}

Received: November 11, 2021

Published: November 29, 2021

(C) All rights are reserved by Susana

Andrade and Jorge Humberto Martins

\begin{abstract}
Objectives: This study aimed to evaluate the benefits of cochlear implant in adults with more than 65 years of age at time of surgery compared with those obtained by adults implanted at younger ages. The author also intended to evaluate the impact of cochlear implantation in the quality of life of older patients.

Results: Outcomes of verbal discrimination performance in elderly cochlear implant users were generally lower than those obtained by the remaining adult population, with a statistical difference in 7 of 11 tests used in the study. The results obtained at the questionnaires answered by the elderly group revealed a significant reduction of hearing handicap after cochlear implantation.

Conclusions: Age alone should not be a contraindication for hearing rehabilitation by cochlear implant. Despite producing a lower performance compared to that achieved by younger patients, cochlear implant in the elderly provides a significant improvement in quality of life.
\end{abstract}

Keywords: Cochlear Implant; Elderly; Hearing Rehabilitation; Quality of Life

\section{Introduction}

The auditory rehabilitation of patients with severe to profound sensorineural hearing loss, with insufficient results through conventional prosthetic amplification, underwent a drastic revolution after the introduction of cochlear implantation (CI). The auditory and psycho-social benefits resulting from $\mathrm{CI}$ have been extensively documented, consolidating this rehabilitation method as an effective and safe technique, promoting a significant improvement in the quality of life and enabling the integration of these patients into an active society.

The global trend of population aging has lately translated into an increase in the number of elderly people seeking CI centers. In fact, since the last half of the 20th century, the geriatric population has assumed an increasing representation in our society, as a result of the increase in average life expectancy. According to data from the "united nations program on ageing" (WHO ageing), 1 in $10 \mathrm{Eu}$ ropeans is over 60 years old. This ratio is expected to be 1:5 in 2050 and 1:3 over the next 100 years. In Portugal, the number of elderly people has doubled in the last four decades, currently representing $16.4 \%$ of the total population. Estimated data published by the national institute of statistics predict that the geriatric population will rise to more than $30 \%$ in the next 40 years [1].

The benefit of cochlear implantation in this group of patients has been the subject of some controversy. Initially, the possible negative influence of age on their auditory performance was considered, justified by the progressive degradation of the peripheral and central auditory systems [2-5] and aggravated by underlying 
cognitive deficits, prolonged time of deafness, learning and communication difficulties. On the other hand, the presence of physical comorbidities could potentially determine a higher risk of anesthetic and surgical complications, as well as prolonging postoperative recovery $[6,7]$.

As a treatment for a non-life-threatening pathology, it was questioned whether cochlear implantation would not be an excessively expensive and unnecessary procedure, when intended for patients of retirement age, and therefore with supposedly low productivity. However, the social and psychological implications of hearing loss in these patients are not negligible, and its impact on health in general is known, causing less mobility and activity, reduced interpersonal contacts and isolation, loss of joy in living and depression [8]. Hearing impairment in the elderly, with all its constraints, should not be underestimated; on the contrary, it should deserve adequate treatment.

Several studies have currently demonstrated that CI is a beneficial procedure for these patients is safe, well tolerated [9-12], improving their auditory performance [13,14], quality of life [15] and CI results in an improvement in cognitive subdomains [16].

This study aims to assess the benefits of cochlear implantation in elderly patients, as well as the impact on their quality of life. Results related to post-cochlear implant auditory performance in this population are compared with those obtained by implanted adults at younger ages.

\section{Material and Methods}

A retrospective and descriptive study was carried out, which included all adult patients with severe to profound post-lingual sensorineural deafness who underwent CI at the Cochlear Implants Unit of the ENT Service of the Hospital Center of Coimbra, in a period between 1985 and 2020, totaling 660 individuals. All patients were implanted after an exhaustive preoperative evaluation, according to the protocol used in this service, in order to determine the clinical indication for $\mathrm{CI}$.

From the study population, patients with a minimum of 6 months of hearing experience with a cochlear implant were selected. They were divided into two groups, considering the age at the time of cochlear implantation surgery: Group I - age $\geq 65$ years; Group II (control) - age $\geq 18$ years and $<65$ years.
Data were collected through the analysis of clinical files and access to computerized medical records, relating to demographic data, audiometric data and results of verbal discrimination tests.

The preoperative audiological data referred to the results of the tonal and vocal audiogram in free field; the postoperative audiometric evaluation included the same exams performed with the cochlear implant.

The analysis of auditory performance was complemented with assessment of verbal discrimination, by presenting different verbal stimuli integrated in 11 tests, using lists of stimuli - monosyllables, numbers and sentences [17], vowels, consonants, 100 words and the pair discrimination test minimum (DPM), no visual cues. In the monosyllables and numbers tests, correctly repeated words and phonemes were counted; in the sentences and 100 words tests, correctly repeated words; in vowels and consonants, correctly repeated or identified stimuli; in the discrimination of minimal pairs, the correctness of the distinction of "equal words" or "different words" was considered. The verbal stimuli included in the lists of 100 words and phrases were also presented over the telephone.

The results obtained were compared between the two study groups. In the statistical analysis, the normality of the sample distribution was verified by the Kolmogorov-Smirnov test. In the absence of normal distribution, the non-parametric test for two independent samples Mann-Whitney U was applied; given normal distribution, the $t$ test for paired samples was used.

Two self-assessment questionnaires based on the Hearing Handicap Inventory for the Elderly (P-HHIE) [18], European Portuguese validated version of the Hearing Handicap Inventory were sent to each patient aged 65 years or over for the Elderly (HHIE) [19]. The $1^{\text {st }}$ questionnaire included questions related to quality of life before HF; the 2nd questionnaire intended that they answer the same questions, but referring to the current situation, after starting the rehabilitation program with cochlear implant. They were also asked about the degree of general satisfaction, daily use and difficulties in handling the speech processor.

\section{Results}

The 588 patients were selected with an overall mean age of 46.7 \pm 15.2 years at the time of cochlear implantation (Table 1 ). 


\begin{tabular}{|l|c|c|}
\hline Descriptive statistics & Group I & Group II \\
\hline Gender & & \\
Male & 17 & 107 \\
Female & 15 & 91 \\
Mean age of implantation (SD), years & $71.9(5.6)$ & $42.5(11.9)$ \\
Minimum age implantation, years & 65 & 18 \\
Maximum age implantation, years & 83 & 64 \\
Implanted Ear & & \\
Right & 18 & 126 \\
Left & 14 & 72 \\
\hline
\end{tabular}

Table 1: Statistical characterization of the sample.

Of these, $13.7 \%(n=93)$ were aged $\geq 65$ years at the time of CI surgery (Group I) and the remaining $86,3 \%(n=85,1)$ were implanted aged $\geq 18$ years and $<65$ years (Group II).

The etiology of deafness was mostly attributed to progressive idiopathic deafness in both groups (Graph 1).

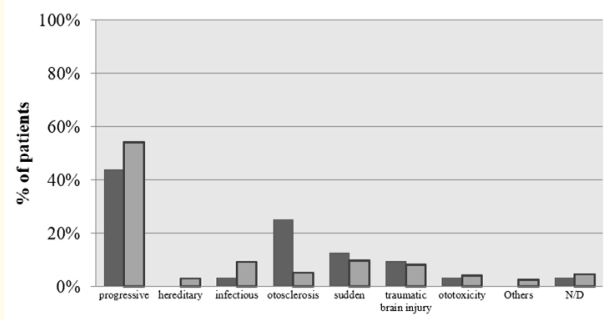

Group $\mathrm{I}(\geq 65$ years) 口Group Il ( $<65$ years $)$

Graph 1: Etiology of deafness in patients in the sample.

The mean preoperative global hearing threshold assessed by free field tonal audiogram was $95.8 \pm 4.6 \mathrm{dBSPL}$ and $96.3 \pm 13.2$ dBSPL, in Groups I and II, respectively, with no statistically significant differences between the groups for $\mathrm{p} \leq 0.05$. An average of $34.7 \pm 7.0 \mathrm{dBSPL}$ and $33.3 \pm 7.9 \mathrm{dBSPL}$ was obtained after $\mathrm{CI}$, for groups I and II, respectively (Figure 1).

Comparing the results of the postoperative audiometric evaluation between the two groups, no statistically significant differences were found, except for the $4000 \mathrm{~Hz}$ frequency ( $p=0.016)$.
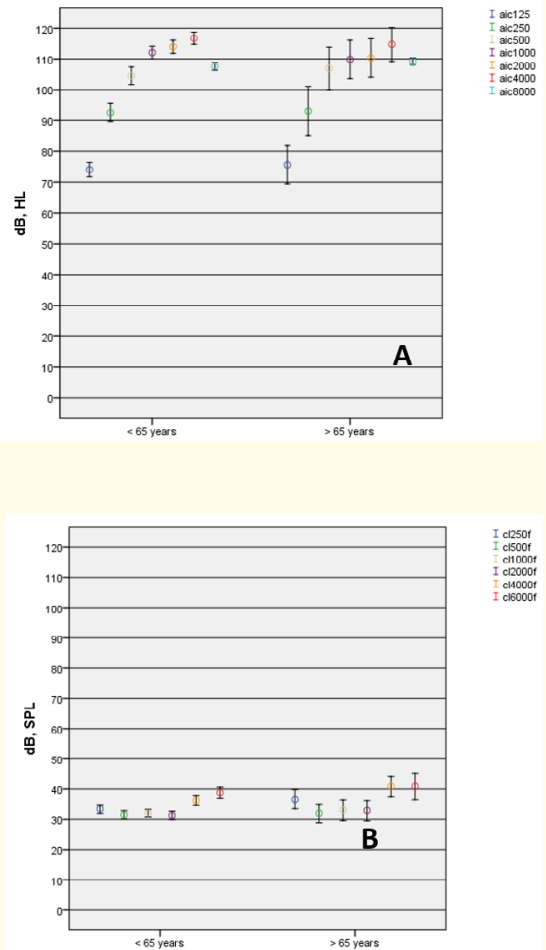

Figure 1: Diagram representing: A. global hearing threshold in free field before CI; B. Global hearing threshold in free field after CI, for each frequency, in Groups I and II.

Regarding the vocal audiogram, the cochlear implant promoted a significant improvement in vocal discrimination in both groups, for each of the tested intensities (Figure 2), with a statistically significant difference between the groups, in the intensities of 60,70 and 80 dBSPL ( $\mathrm{p} \leq 0.05)$.

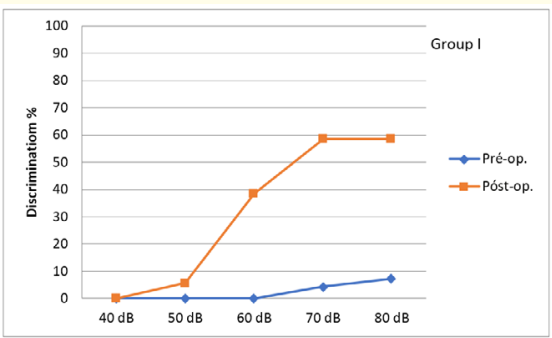




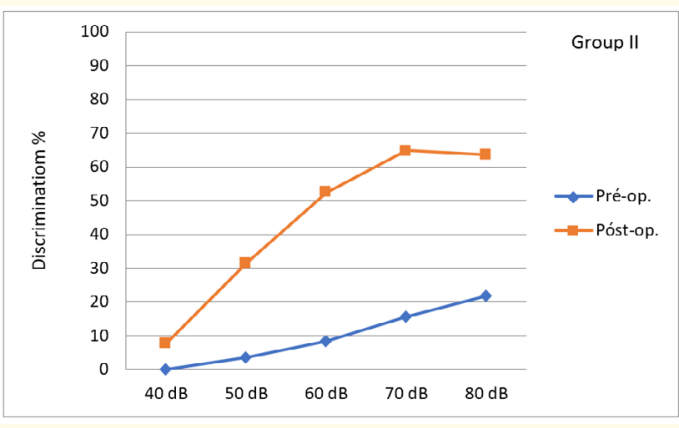

Figure 2: Speech audiometry - comparison between preoperative and postoperative results for Group I and Group II.

The results of post-cochlear implantation auditory performance, expressed as the percentage of elements correctly answered in each list of the test battery used.

In general, the average performance of individuals in Group II is better than the performance achieved by individuals in Group I. In tests where the requested task involves simpler linguistic material, as in the case of number tests (numbers and phonemes) and vowels, this difference is frankly attenuated. The opposite is observed in tests that imply a greater complexity of the task, namely the tests of phrases and 100 words on the phone. Tests with presentation of elements under telephone conditions presented, in both groups, a greater degree of difficulty compared to those performed in person.

In the discrimination tests in which lists of numbers, vowels, consonants and the DPM test were applied, no statistically significant differences were found between groups I and II $(\mathrm{p} \leq 0.05)$. The remaining results were statistically different.

The analysis of the correlation of the variable "result of verbal discrimination" with the variable "age to cochlear implantation data", for each of the tests and considering the entire study sample, was preceded by the performance of the Kolmogorov-Smirnov test to analyze the normality of this sample.

There was only a normal distribution of the sample in the consonant test, so the measurement of the intensity of the relationship between the two variables in question was based on the determination of Spearman's Ró correlation coefficient, which does not require the assumption of normal distribution Sample (Table 2).

\begin{tabular}{|l|c|c|c|c|}
\hline \multicolumn{2}{|c|}{} & $\begin{array}{c}\text { Duration of } \\
\text { Deafness }\end{array}$ & $\begin{array}{c}\text { Sentences } \\
\text { test (\%) }\end{array}$ \\
\hline \multirow{4}{*}{$\begin{array}{l}\text { Suration of } \\
\text { Spearman's } \\
\text { Row }\end{array}$} & $\begin{array}{c}\text { Correlation } \\
\text { coeficiente }\end{array}$ & 1,000 &,- 324 \\
\cline { 3 - 5 } & Deafness & Sig. (2-tailed) &. &, 000 \\
\cline { 3 - 5 } & \multirow{2}{*}{$\begin{array}{c}\text { Sentences } \\
\text { test (\%) }\end{array}$} & $\begin{array}{c}\text { Correlation } \\
\text { coeficiente }\end{array}$ &,- 324 & 1,000 \\
\cline { 3 - 5 } & Sig. (2-tailed) &, 000 & $\cdot$ \\
\cline { 3 - 5 } & & $\mathrm{N}$ & 137 & 197 \\
\hline
\end{tabular}

Table 2: Spearman's Rho correlations.

Scatter plots were created with individual results in each of the tests as a function of age and CI data, followed by correlation analyzes for the total sample and for each of the groups, following the same procedure.

In the MNS tests, it was verified the existence of a negative association between the variables, especially in the first test, except for an exceptional positive association of group II in the discrimination of phonemes. This association was of weak intensity (correlation coefficient close to "0") and does not signify any of the lists (Graph 3).

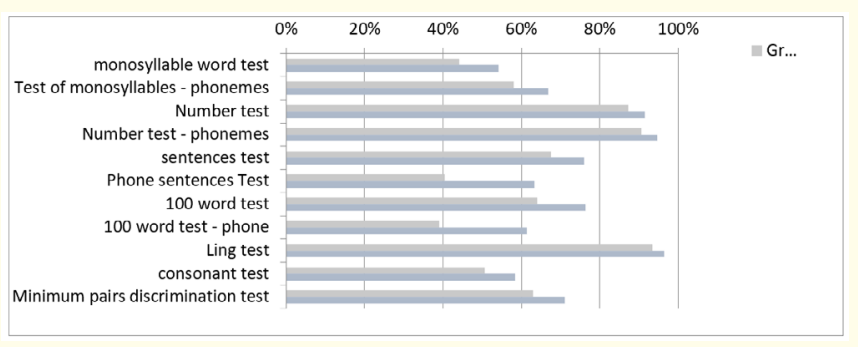

Graph 2: Average values obtained in the post-CI auditory discrimination tests in both groups. 


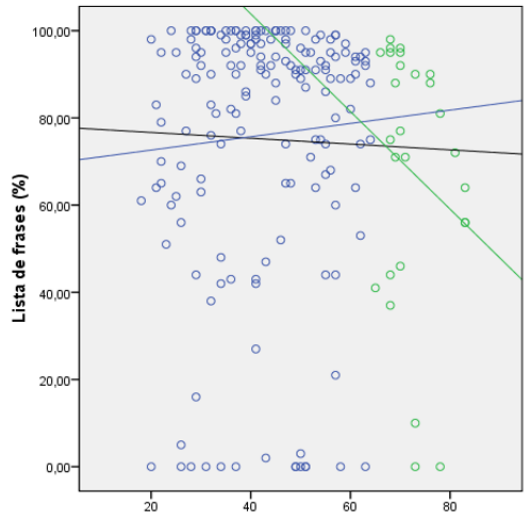

Graph 3: The Graph shows the relationship between the variable "Phrase Test" and the age of implantation.

In the number tests, there was a negative association between the variables, with the exclusion of Group II in the discrimination of phonemes. In any group of elderly patients, the correlation was stronger in both testes than in younger patients. The results did not reach statistical significance.

There was a negative association between the implementation and discrimination of sentences, only statistically significant in Group II regarding discrimination on the telephone. In elderly patients, the most pronounced degree of association corresponded to the test in which the stimulus was presented by telephone (Spearman's Rho $=0.341$ ), although without statistical significance.

In the 100 -word tests, a weak statistically significant negative association was also observed in the total sample. When equal groups eliminate, this significance is not verified.

A negative association between the variables was verified in the vowel tests, when the total sample and group I were analyzed. In group II, a positive association was found. Non-global correlation coefficients translate to an association of very weak intensity. In the consonant test, the negative association, although weak, was statistically significantly for the total sample $(p=0.043)$; the correlation in the involved groups has not been defined.

In the matched pair discrimination test, the association was also weak, negative, and unrestricted.
Each patient belonging to Group I was sent a pair of P-HHIE questionnaires. We got responses from 19 patients.

There was a decrease in the total score after cochlear implantation, with a statistically significant decrease in self-perception of hearing handicap, an indication of an improvement in quality of life. All patients use the cochlear implant daily and 68.5\% report using the telephone. $15.8 \%$ of the elderly implanted occasionally need help handling the processor. All patients questioned would recommend the Cochlear Implant to a friend who suffered from the same hearing difficulty.

\section{Discussion}

The age distribution of patients implanted in the CHC's ENT Service, over a trajectory of 35 years of experience and similarly to other cochlear implantation centers, reflects a growing integration of the elderly in hearing rehabilitation programs for $\mathrm{CI}$, accompanied by some shape the demographic changes observed in recent years.

In this study, most of the results obtained in tests of verbal perception were slightly lower in the group of elderly patients compared to those observed in adults implanted in younger age groups, in line with some published studies $[7,20]$. Despite this, the use of cochlear implants resulted in very satisfactory results in the elderly population, in terms of discrimination, a fact that is very evident in the postoperative vocal audiogram [12].

Although the two groups were audiologically similar in terms of tonal perception in both the preoperative and postoperative assessments, the difference in performance in the speech perception tests between the two groups was statistically significant in 7 of the 11 tests applied in this study. The degradation of the peripheral auditory system inherent to age, due to mechanisms of loss of sensory hair cells [21], reduction of cochlear neuronal populations and atrophy of the stria vascularis [4], is not theoretically an impediment to obtaining good results with cochlear implantation, since this procedure allows for direct stimulation of the auditory nerve fibers. This difference in the ability of verbal discrimination has been attributed to deficits in central auditory processing related to physiological changes in the central auditory pathways [2,4], which are also inherent to age, combined with a lower capacity for attention, learning and communication [22]. In fact, the tonal hearing benefit may not be accompanied by a proportional gain in dis- 
crimination [23]. Verbal discrimination assessed by speech perception tests involves more complex auditory processing mechanisms, dependent on the associated auditory areas located at the level of the upper cortex, and therefore more susceptible to age-related deterioration phenomena, contrary to tonal detection mechanisms. located in the primary auditory areas.

Nevertheless, several publications have proven $[10,15,24]$ that elderly patients reach a level of performance that is statistically similar to younger adults.

The responses obtained in the self-assessment questionnaire suggest that cochlear implantation in the elderly provides a subjective improvement in social life, self-confidence and quality of life in general, allowing them a more fulfilled and interactive existence, in line with results reported in previous publications [10]. Although the questionnaires were not applied to the remaining sample, several other studies show that elderly patients report an improvement in their quality of life with the cochlear implant, without statistically significant differences compared to younger populations $[11,25]$.

\section{Conclusion}

Cochlear implant hearing rehabilitation determines, in the geriatric population, not only a better hearing performance, but above all a better quality of life, boosting and motivating their independence, activity and social interaction.

\section{Bibliography}

1. Portal de Estatísticas Oficiais - Destaques. Portal do Instituto Nacional de Estatística. Estatística, I.N.d. Portal do Instituto Nacional de Estatística (2012).

2. Welsh LW., et al. "Central presbycusis Welsh". Laryngoscopy 95 (1985): 128-136.

3. Gatehouse $\mathrm{S}$ "The contribution of central auditory factors to auditory disability". Acta Oto-Laryngologica 476 (1990): 182188.

4. HF Schuknecht., et al. "Cochlear pathology in presbycusis". The Annals of Otology, Rhinology, and Laryngology 102 (1993): 1-16.

5. Cheesman MF., et al. "Comparison of grouth of masking functions and speech descrimination abilities in younger and older adults". Audiology 15.4 (1995): 321-333.
6. Muravchick S. "The ageing process: anesthetic implications". Acta Anaesthesiologica Belgica 49 (1995): 85-90.

7. Chatelin V., et al. "Cochlear Implant Outcomes in the Elderly". Otology and Neurotology 25 (2004): 298-301.

8. Herbst KRG. "Psychological consequences of disorders of hearing in the elderly". Hearing and Balance in the Elderly (1983).

9. Pasanini E., et al. "Speech recognition in elderly cochlear implant recipients”. Clinical Otolaryngology 28 (2003): 154-157.

10. Buchman C., et al. "Cochlear implants inthe geriatric population: benefits outweigh risks". Ear Nose Throat (1999): 489494.

11. Eshraghi AA., et al. "Cochlear Implant Surgery in Patients more than seventy-nine years old". Laryngoscopy (2019): 1-4.

12. Yang $\mathrm{Z}$ and Cosetti M. "Safety and outcomes of cochlear implantation in the elderly: A review of recent literature". Journal of Otology 11.1(2016): 1-6.

13. Filipo R., et al. "Cochlear implantation in elderly: indication and results". Catanzaro: BioMed Central (2010).

14. Lundin K., et al. "Cochlear implantation in the elderly". Cochlear Implants International 14.2 (2017):92-97.

15. Poissant S., et al. "Impact of cochlear implantation on speech understanding, depression, and loneliness in the elderly". Journal of Otolaryngology-Head and Neck Surgery 37.4 (2008): 488-494.

16. Issing C., et al. "Impact of Hearing Rehabilitation Using Cochlear Implants on Cognitive Function in Older Patients". Otology and Neurotology 42.8 (2021): 1136-1141.

17. Martins JH., et al. "Validação de listas de monossílabos, números e frases para audiometria vocal para adultos". Clínica e Investigação em Otorrinolaringologia 3.1 (2009): 54-49.

18. Martins Julieta J., et al. "Pontifícia Deficiência Auditiva: Sua Percepção. Universidade Católica de São Paulo". Audiologia em Revista n. 3 (2010): 96-112.

19. Ventry IM and Weinstein BE. "The hearing handicap inventory for the elderly: a new tool”. Ear Hear 3.3 (1982): 128-134.

20. Runge-Samuelson C., et al. "Case-control Analysis of Cochlear Implant Performance in Elderly". Archives of Otorhinolaryngology-Head and Neck Surgery 136.5 (2010): 432-438. 
21. Nadol JB. "Electron microscopic findings in presbycusic degeneration of the basal turn of the human cochlea". Otorhinolaryngology-Head and Neck Surgery 87 (1979): 818-836.

22. Mahnke CD., et al. "Brain plasticity and functional losses in the age: scientific bases for a novel intervention". Progress in Brain Research (2006): 81-109.

23. Leung J., et al. "Predictive Models for Cochlear Implantation in Elderly Candidates". Archives of Otorhinolaryngology-Head and Neck Surgery 131 (2005): 1049-1054.

24. Carlson Mathew., et al. "Cochlear implant". Otology and Neurotology 31 (2010): 1343-1349.

25. Djalilian HR., et al. "Cochlear implantation in the elderly: results and quality-of-life assessment". The Annals of Otology, Rhinology, and Laryngology 111 (2002): 890-895.

Volume 3 Issue 12 December 2021

(c) All rights are reserved by Susana Andrade and Jorge Humberto Martins. 